\section{ON THE CONTINUATION}

\section{OF HOLOMORPHIC MAPPINGS}

Mamoru Yoshida*

\section{Introduction}

In the theory of one complex variable, it is well known that holomorphic functions cannot in general be extended as holomorphic functions to larger domains. For example, if $\Omega(\neq C)$ is open and $a \in \partial \Omega$, then

$$
f(z)=\frac{1}{z-a}
$$

cannot be extended across the boundary point $a$. Another classical example is

$$
g(z)=\Sigma_{n=1}^{\infty} z^{n !}
$$

which cannot be extended across any point of the boundary of the unit disc. For any domain $\Omega$ of $\mathrm{C}$, there is a holomorphic function which cannot be extended across any point of the boundary $\partial \Omega$.

For functions of two variables, we have a new phenomenon. For example if we consider the annulus

$$
\Omega_{1}=\left\{\left(z_{1}, z_{2}\right): 1<\left|z_{1}\right|^{2}+\left|z_{2}\right|^{2}<4\right\},
$$

then all $f$ holomorphic on $\Omega$ can be extended to the ball

$$
\tilde{\Omega}_{1}=\left\{\left(z_{1}, z_{2}\right):\left|z_{1}\right|^{2}+\left|z_{2}\right|^{2}<4\right\} .
$$

A simple consequence of this result is that zeros of holomorphic functions of more than one variable cannot be isolated.

* The author thanks University College Dublin for support during the period this research was undertaken.
Another classical example concerns the domain

$$
\Omega_{2}=\left\{\left(z_{1}, z_{2}\right):\left|z_{1}\right|<1,\left|z_{2}\right|<1,|| z_{1}|-| z_{2}||<\frac{1}{3}\right\} .
$$

All $f$ holomorphic on $\Omega_{2}$ can be extended to the polydisc

$$
\tilde{\Omega}_{2}=\left\{\left(z_{1}, z_{2}\right):\left|z_{1}\right|<1,\left|z_{2}\right|<1\right\} .
$$

When this phenomenon, which was discovered by Hartogs, occurs, it is natural to ask the following questions:

(A) Given $f$ holomorphic on $\Omega$, is there a natural domain to which

all $f$ holomorphic on $\Omega$ can be extended holomorphically ?

(B) Given $\Omega$, is there a natural domain $\Omega^{\prime}$ to which all holomorphic functions on $\Omega$ can be extended?

If a natural domain for $f$ exists, we call such a domain (in (A)) a domain of existence of $f$ and denote it by $\Omega_{f}$. For example the unit disc in $\mathbb{C}$ is the natural domain of existence of $g(z)=\Sigma_{n=1}^{\infty} z^{n !}$.

Is $\Omega_{f}$ unique? How does one construct $\Omega_{f}$ ?

Such a natural domain $\Omega^{\prime}(\mathrm{in}(\mathrm{B}))$ is called a domain of holomorphy.

It is natural to expect that, in some sense, $\Omega^{\prime}$ is related to $\bigcap_{f \in \mathcal{H}(\Omega)} \Omega_{f}$ where $\mathcal{H}(\Omega)$ is the family of all holomorphic functions in $\Omega$. Is it possible to characterize when $\Omega=\Omega^{\prime}$ geometrically or algebraically?

\section{Geometric characterization}

The proper mathematical definitions are rather technical but we can state some results which are readily understandable. For example the following notion of holomorphically convex domain is related, by the Hahn-Banach Theorem, to the linear notion of convexity.

Let $\Omega$ be a domain of $C^{n}$ and $K$ any compact subset in $\Omega$. We put

$$
\hat{K}_{\Omega}=\left\{z \in \Omega:|f(z)| \leq\|f\|_{K}, f \in \mathcal{H}(\Omega)\right\}
$$

where $\|f\|_{K}=\sup \{|f(z)|: z \in K\}$. 
If $\hat{K}_{\Omega}$ is also compact for any $K, \Omega$ is said to be holomorphically convex. It is easily seen that convex domains are holomorphically convex.

We have the following two equivalent properties for a domain $\Omega$ of $\mathbf{C}^{n}$ :

(I) $\Omega$ is a domain of holomorphy,

(II) $\Omega$ is holomorphically convex.

\section{Metric characterization}

A function $\mu$ on a connected domain $D(\subset \mathrm{C})$ is called subharmonic, if the following conditions are satisfied:

(1) $\mu$ is upper semicontinuous,

(2) $\mu$ is not identically $-\infty$

(3) $\mu(c) \leq \frac{1}{2 \pi} \int_{0}^{2 \pi} \mu\left(c+r e^{i \theta}\right) d \theta, \quad(r>0$ : sufficiently small $)$.

Condition (1) is a smoothness condition, while (2) is just to exclude a trivial case and (3) is the main property which says that the average over a disc dominates the value at the centre.

Let $\Omega$ be a connected domain of $\mathrm{C}^{n}$ and let $z_{0}$ be any point in $\Omega$. We put

$$
E_{a}\left(z_{0}\right)=\left\{z=z_{0}+a t: t \in \mathbb{C}\right\},\left(a \in \mathbb{C}^{n}\right) .
$$

If $\mu$ is not identically $-\infty$ and $\mu$ satisfies (1) and (3) as a function of $t$ in $\Omega \cap E_{a}\left(z_{0}\right)$ for all $z_{0} \in \Omega$ and $a \in \mathbf{C}^{n}$, then $\mu$ is said to be plurisubharmonic on $\Omega$.

If $-\log d(z, \mathrm{C} \Omega)$ is plurisubharmonic in $\Omega$, then $\Omega$ is called pseudoconvex.

The following condition for a domain $\Omega$ of $\mathbf{C}^{n}$ is equivalent to conditions (I) and (II) above:

(III) $\Omega$ is pseudoconvex.

It is not difficult to show that domains of existence are pseudoconvex. The converse was conjectured by E. E. Levi in 1911 and became known as the Levi problem and was solved by K. Oka in 1942 for domains in $\mathrm{C}^{2}$ and for domains in $\mathrm{C}^{n}$ by F. Norguet and $H$. Bremermann in 1954. The term 'Levi problem' (see section 2) is still used for problems of this type in more abstract and general settings.

\section{Algebraic characterizations}

For a given family (data) of meromorphic functions which are defined locally on a domain $\Omega$ of $\mathbf{C}^{n}$, can we find a global meromorphic function which is locally identified, in a sense, by the data? More precisely given an open covering $\mathcal{U}=\left\{U_{i}: i \in\right.$ $I\}$ of $\Omega$ and a collection of meromorphic functions $m_{i}$, with $m_{i}$ meromorphic (i.e. a quotient of holomorphic functions) on $U_{i}$ Supposing that $m_{i}-m_{j}$ is holomorphic on $U_{i} \cap U_{j}$, then we wish to find a global meromorphic function $m$ on $\Omega$ such that $m-m_{i}$ is holomorphic on $U_{i}$ for all $i$. Let $\mathcal{O}$ be the sheaf of germs of holomorphic functions over a domain $\Omega$ of $\mathbf{C}^{n}$. If a collection $Z^{1}(\Omega, \mathcal{O}):=\left\{z_{i j}: i, j \in I\right\}$ satisfies conditions (1), (2) and (3) below, we call $Z^{1}(\Omega, \mathcal{O})$ a holomorphic cocycle of degree one on $\mathcal{U}$ :

(1) $z_{i j}$ is holomorphic on $U_{i} \cap U_{j}(\neq \emptyset)$ for all $i$ and $j$ in $I$,

(2) $z_{i j}=-z_{j i}$ on $U_{i} \cap U_{j}$ whenever $U_{i} \cap U_{j} \neq \emptyset$,

(3) $z_{i j}+z_{j k}+z_{k i}=0$ on $U_{i} \cap U_{j} \cap U_{k}$ whenever $U_{i} \cap U_{j} \cap U_{k} \neq \emptyset$. If $C^{1}(\Omega, \mathcal{O}):=\left\{z_{i}: i \in I\right\}$ satisfies condition (4) below, we call $C^{1}(\Omega, \mathcal{O})$ a holomorphic cochain of degree one on $\mathcal{U}$ :

(4) $z_{i}$ is holomorphic on $U_{i}$ for all $i \in I$. We put

$$
B^{1}(\Omega, \mathcal{O})=\left\{z_{i}-z_{j}: z_{i}, z_{j} \in C^{1}(\Omega, \mathcal{O})\right\}
$$

and call $B^{1}(\Omega, \mathcal{O})$ a holomorphic coboundary of degree one on $\mathcal{U}$. $Z^{1}(\Omega, \mathcal{O})$ and $B^{1}(\Omega, \mathcal{O})$ are additive groups and $B^{1}(\Omega, \mathcal{O})$ is a subgroup of $Z^{1}(\Omega, \mathcal{O})$.

We put $H^{1}(\Omega, \mathcal{O})=Z^{1}(\Omega, \mathcal{O}) / B^{1}(\Omega, \mathcal{O})$ and call $H^{1}(\Omega, \mathcal{O})$ the first holomorphic cohomology group on $\mathcal{U}$.

Clearly $H^{1}(\Omega, \mathcal{O})=0$ if and only if $Z^{1}(\Omega, \mathcal{O})=B^{1}(\Omega, \mathcal{O})$.

The following condition is equivalent to $(\mathrm{I})$ :

(IV) $H^{1}(\Omega, \mathcal{O})=0$.

A very different algebraic characterization has been investigated by other authors. If $\mathcal{H}(\Omega)$ is endowed with the compact open topology, then it becomes a complete metrizable topological algebra (i.e. the operations of addition and multiplication are continuous). We may then consider $\mathcal{M}(\mathcal{H}(\Omega))$, the set of all continuous $\mathrm{C}$-valued multiplicative linear functions on $\mathcal{H}(\Omega)$. Clearly point evaluations belong to $\mathcal{M}(\mathcal{H}(\Omega))$ and thus the the mapping

$$
\varphi: x \in \Omega \rightarrow \delta_{x} \in \mathcal{M}(\mathcal{H}(\Omega)) \text {, }
$$


where $\delta_{x}$ is point evaluation at $x$, is well defined and easily seen to be injective for $\Omega$ open in $\mathbb{C}^{n}$. The space $\mathcal{M}(\mathcal{H}(\Omega))$ can be endowed with the structure of a complex manifold and with this structure the envelope of holomorphy of $\Omega$ can be identified with the connected component of $\mathcal{M}(\mathcal{H}(\Omega)$ ) which contains $\varphi(\Omega)$. This gives the following characterization equivalent to condition (I) for a domain $\Omega$ of $\mathrm{C}^{n}$

(V) $\Omega=\mathcal{M}(\mathcal{H}(\Omega))$.

Conditions (II) and (III) are geometric characterizations of domains of holomorphy while (IV) and (V) are algebraic characterizations. In particular, (II) reduces to whether $-\log d(z, \mathrm{C} \Omega)$ is subharmonic on a domain of the complex plane $\mathrm{C}$. Condition (IV) is suitable for concrete calculations.

These are parts of the basic classical background to more recent results which we will now describe. The results we describe involve a more general setting.

\section{Setting up of the problem}

We describe the more general setting.

(1) First it is more natural to discuss such problems over Riemann domains than open sets of $\mathbb{C}^{n}$, since for example there exists an open subset of $\mathrm{C}^{n}, \Omega$, say, such that holomorphic func. tions on $\Omega$ cannot be extended to any larger open subset of $\mathrm{C}^{n}$, but if $\Omega$ is considered as a complex manifold, then all holomorphic functions on $\Omega$ can be extended to a strictly larger complex manifold.

The definition of Riemann domain is as follows:

if there exists a local biholomorphic mapping $\psi$ of a complex manifold $\Omega$ into the locally convex space $E,(\Omega, \psi)$ is called a Riemann domain over $E$.

(2) We discuss Riemann domains over infinite dimensional spaces rather than over $\mathbf{C}^{n}$. In this case a mapping is holomorphic if it is continuous and its restriction to each finite dimensional section is holomorphic as a mapping of several complex variables.

(3) We discuss mappings of a Riemann domain $\Omega$ into a complex manifold $N$.

(4) We consider subsets $\mathcal{F}$ of the space of all holomorphic mappings. The two simplest examples are $\mathcal{F}=\mathcal{H}(\Omega)$ which leads to the concept of domain of holomorphy and $\mathcal{F}=\{f\}$ (i.e. $\mathcal{F}$ consists of a single function $f$ ) and this leads to the concept of domain of existence. We confine ourselves here to these two examples but mention a further natural and useful example, $\mathcal{H}^{\infty}(\Omega)$-the space of bounded holomorphic functions on $\Omega$. Note that if $\Omega \subset \Omega^{\prime}$ and each holomorphic function on $\Omega$ extends, as a holomorphic function, to $\Omega^{\prime}$ then if $f \in \mathcal{H}(\Omega), \lambda \in \mathbb{C}$ and $\lambda \notin f(\Omega)$ then

$$
g:=\frac{1}{f-\lambda} \in \mathcal{H}(\Omega)
$$

and hence admits a holomorphic extension $\tilde{g}$ to $\Omega^{\prime}$. By uniqueness of holomorphic extensions it follows that $(f-\lambda) \cdot g=1$ on $\Omega$ and $(\tilde{f}-\lambda) \cdot \tilde{g}=1$ on $\Omega^{\prime}$, where $\tilde{f}$ is the extension of $f$ to $\Omega^{\prime}$. Hence $\lambda \notin \tilde{f}\left(\Omega^{\prime}\right)$ and it is now easy to see that whenever $f \in \mathcal{H}^{\infty}(\Omega)$ then $\tilde{f} \in \mathcal{H}^{\infty}\left(\Omega^{\prime}\right)$.

Next we provide some terminology for mappings between Riemann domains in order to set up our problem precisely. Let $(\Omega, \psi)$ and $\left(\Omega^{\prime}, \psi^{\prime}\right)$ be Riemann domains over a local convex space $E$. If a holomorphic mapping $\lambda$ of $\Omega$ into $\Omega^{\prime}$ satisfies $\psi=\psi^{\prime} \circ \lambda$, the mapping $\lambda$ is called a morphism of $(\Omega, \psi)$ into $\left(\Omega^{\prime}, \psi^{\prime}\right)$. Let $N$ be a complex manifold and let $\mathcal{F} \subset \mathcal{H}(\Omega, N)$, where

$$
\mathcal{H}(\Omega, N)=\{f: f \text { is a holomorphic mapping of } \Omega \text { into } N\} \text {. }
$$

A morphism $\lambda$ of $(\Omega, \psi)$ into $\left(\Omega^{\prime}, \psi^{\prime}\right)$ is said to be an $\mathcal{F} N$ extension of $\Omega$ if for each $f \in \mathcal{F}$ there exists a unique $f^{\prime} \in$ $\mathcal{H}\left(\Omega^{\prime}, N\right)$ such that $f^{\prime} \circ \lambda=f$. When $\mathcal{F}=\mathcal{H}(\Omega)$ and $N=\mathbb{C}$ we simply say a holomorphic extension.

$\Omega$ is said to be an $\mathcal{F} N$-domain of holomorphy if each $\mathcal{F N}$ extension of $\Omega$ is an isomorphism.

$\Omega$ is said to be a domain of holomorphy if each holomorphic extension is an isomorphism.

$\Omega$ is said to be a domain of existence if there exists $f \in \mathcal{H}(\Omega)$ such that $\Omega$ is an $\{f\}$ C-domain of holomorphy. 
Let $(\Omega, \psi)$ be a Riemann domain over the space $E$ and let $\mathcal{F} \subset \mathcal{H}(\Omega, N)$. A morphism $\lambda: \Omega \rightarrow \Omega^{\prime}$ is called an $\mathcal{F} N$-envelope of holomorphy of $\Omega$ if:

(a) $\lambda$ is an $\mathcal{F} N$-extension of $\Omega$,

(b) if $\mu: \Omega \rightarrow \Omega^{\prime \prime}$ is an $\mathcal{F} N$-extension of $\Omega$, then there exists a morphism $\nu: \Omega^{\prime \prime} \rightarrow \Omega^{\prime}$ such that $\nu \circ \mu=\lambda$.

Then $\left(\lambda, \Omega^{\prime}, \psi^{\prime}\right)$ or $\Omega^{\prime}$ is also called $\mathcal{F} N$-envelope of holomorphy of $(\Omega, \psi)$ or $\Omega$. Note that condition (b) says that an $\mathcal{F} N$ extension is maximal.

Malgrange, [10, pp.29-34] for the finite dimensional case and Mujica, [11, Theorem 56.4] for the infinite dimensional case, proved that there exists an $\mathcal{F} N$-envelope of holomorphy of $\Omega$ and that this envelope is unique up to complex analytic isomorphism.

Let $(\Omega, \psi)$ be a Riemann domain over the space $E,(\tilde{\lambda}, \tilde{\Omega}, \tilde{\psi})$ its envelope of holomorphy and $N$ a complex manifold.

Problem (*) : Can every $f \in \mathcal{H}(\Omega, N)$ be extended holomorphically to an element $\tilde{f}$ of $\mathcal{H}(\tilde{\Omega}, N)$ such that $f=\tilde{f} \circ \tilde{\lambda}$ ?

\section{Some results}

We require certain restrictions on $N$. If $N=\mathrm{C}_{\cdot, \text { it }}^{n}$ is clear that there is a function $\tilde{f}$ which solves Problem (*). (See, for example, [7].)

If the complex manifold $G$ is a group and the mappings $G \times$ $G \ni(x, y) \rightarrow x \cdot y \in G$ and $G \ni x \rightarrow x^{-1} \in G$ are holomorphic, we call $G$ a complex Lie group. If $\mathcal{G}$ is the tangent space at the identity $e \in G$, we call $\mathcal{G}$ the Lie algebra of $G$. For example, the complex general linear group $G L(n, \mathrm{C})$ consisting of all invertible $n \times n$ complex matrices is a complex Lie group and the set of all matrices of degree $n, \operatorname{gl}(n, \mathbf{C})$, is the Lie algebra with respect to the commutator product $[A, B]=A B-B A$. Between a complex Lie group $G$ and its Lie algebra $\mathcal{G}$, there exists an exponential mapping $\exp : \mathcal{G} \rightarrow G$ which is a local biholomorphism of a neighbourhood of 0 in $\mathcal{G}$ onto a neighbourhood of the identity $e$ in $G$. In the example above,

$$
\exp (A)=I_{n}+A+\frac{1}{2 !} A^{2}+\frac{1}{3 !} A^{3}+\cdots \cdots
$$

for any $A \in \operatorname{gl}(n, \mathbb{C})$, where $I_{n}$ is the identity matrix.

If $m=\operatorname{dim} G<\infty$, we may take $\mathcal{G}$ as $\mathbb{C}^{m}$ and we consider a complex Lie group $G$ of positive dimension as a complex manifold. Let $(\Omega, \psi)$ be a Riemann domain over a Stein manifold $S$, $(\tilde{\lambda}, \tilde{\Omega}, \tilde{\psi})$ its envelope of holomorphy, $G$ a complex Lie group and $f$ a holomorphic mapping of $\Omega$ into $G$.

Let $\left(\lambda, \Omega^{\prime}, \psi^{\prime}\right)$ be the $\{f\} G$-envelope of holomorphy. By pseudoconvexity of $\left(\Omega^{\prime}, \psi^{\prime}\right)$ and the positive solution of DocquierGravert, [3], to the Levi problem, $\left(\Omega^{\prime}, \psi^{\prime}\right)$ is a domain of existence of a holomorphic function on $\Omega$. Since $(\tilde{\lambda}, \tilde{\Omega}, \tilde{\psi})$ is its envelope of holomorphy, there is a mapping $\mu$ of $(\tilde{\Omega}, \tilde{\psi})$ into $\left(\Omega^{\prime}, \psi^{\prime}\right)$ such that $\lambda=\mu \circ \tilde{\lambda}$. Let $f^{\prime}$ be the holomorphic continuation of $f$ to $\left(\lambda, \Omega^{\prime}, \psi^{\prime}\right)$. Then $f^{\prime} \circ \mu$ is the holomorphic continuation of $f$ to $(\tilde{\lambda}, \tilde{\Omega}, \bar{\psi})$. Thus we obtain the following theorem.

Theorem 1. Let $(\Omega, \psi)$ be a Riemann domain over a Stein manifold $S$ and $(\tilde{\lambda}, \tilde{\Omega}, \tilde{\psi})$ its envelope of holomorphy. Any holomorphic mapping of $\Omega$ into a complex Lie group $G$ has a holomorphic continuation to $(\tilde{\lambda}, \tilde{\Omega}, \tilde{\psi})$.

Let $E$ be a complex linear space with the finite open topology $T_{0}$. S. Dineen, [4], proved the vanishing theorem $H^{1}(D, \mathcal{O})=0$ for $D$ pseudoconvex and $\mathcal{O}$ the structural sheaf over the $\mathrm{C}$-linear space $\left(E, T_{0}\right)$ and L. Gruman, [5], solved the Levi problem by proving that any finitely pseudoconvex domain $D$ of the space $\left(E, T_{0}\right)$ is the domain of existence of a holomorphic function on $D$.

A complex manifold $N$ is called a complex Banach manifold if it is a complex manifold modelled on a complex Banach space.

A Banach complex Lie group is a group $G$ which is a complex Banach manifold and a complex Lie group. a set

Let $\mathcal{U}=\left\{U_{i}: i \in I\right\}$ be an open covering of $E$. Assume that

$$
\mathcal{Z}:=\left\{z_{i j}: z_{i j} \in \mathcal{H}\left(U_{i} \cap U_{j}, G\right), i, j \in I\right\}
$$

satisfies conditions similar to conditions $(1),(2)$ and (3) for a holomorphic cocycle of degree one $Z^{1}(\Omega, \mathcal{O})$ which we gave in our algebraic characterization of domains of holomorphy. We call $\mathcal{Z}$ a holomorphic cocycle on $\mathcal{U}$ with values in $G$ and call the pair 
$F=\{\mathcal{U}, \mathcal{Z}\}$ a holomorphic principal fibre bundle with base space $E$ and structure group $G$.

We consider the continuation of holomorphic mappings of a Riemann domain $(\Omega, \psi)$ over $\left(E, T_{0}\right)$ into a complex Banach Lie group $G$. Let $\Lambda$ be the set of finite dimensional $C$-linear subspaces of $E$. For any $L \in \Lambda$, we denote $\Omega \cap \psi^{-1}(L)$ and $\psi \mid \Omega \cap \psi^{-1}(L)$, by $\Omega_{L}$ and $\psi_{L}$, respectively. Any holomorphic mapping $f$ of $\left(\Omega_{L}, \psi_{L}\right)$ into $G$ can be continued holomorphically to its envelope of holomorphy by the finite dimensional result. The authors, [6], proved the following theorem, using the method of Kajiwara-Shon, [9], and transfinite induction as in Dineen, [4].

Theorem 2. Let $E$ be a complex linear space with the finite open topology, $(\Omega, \psi)$ a Riemann domain over $E$ and $(\tilde{\lambda}, \tilde{\Omega}, \tilde{\psi})$ the envelope of holomorphy of $(\Omega, \psi)$. Let $G$ be a complex Banach Lie group. Then any holomorphic mapping of $\Omega$ into $G$ can be holomorphically continued to the envelope of holomorphy $(\tilde{\lambda}, \tilde{\Omega}, \tilde{\psi})$ of $(\Omega, \psi)$.

The main problem with the finite open topology is that it allows of too many holomorphic functions. In two cases in which the base space $E$ is endowed with stronger topologies, the authors; [12], prove, using positive results of Schottenloher, [13], for the Levi problem and methods similar to those used in finite dimension, the following theorem.

Theorem 3. Let $E$ be a separable Fréchet space with the bounded approximation property or a DFN-space and $(\Omega, \psi)$ a Riemann domain over the space $E$. Let $G$ be a complex Banach Lie group and $F$ a holomorphic principal fibre bundle with structure group $G$ over $E$. Then a holomorphic section $s$ of $F$ over $\Omega$ can be continued holomorphically to the envelope of holomorphy of the Riemann domain $\Omega$.

The proofs of the results above are rather technical and so we do not provide details but give one brief example of the technical aspects. Let

$$
D=\left\{z=\left(z_{1}, z_{2}\right) \in \mathbf{C}^{2}:\left|z_{1}\right|<1+\varepsilon,\left|z_{2}\right|<1\right\} \cup
$$

and

$$
\left\{z=\left(z_{1}, z_{2}\right) \in \mathbb{C}^{2}: 1-\varepsilon<\left|z_{1}\right|<1+\varepsilon_{9}\left|z_{2}\right|<1+\varepsilon\right\}
$$

$$
\hat{D}=\left\{z=\left(z_{1}, z_{2}\right) \in \mathbb{C}^{2}:\left|z_{j}\right|<1+\varepsilon(j=1,2)\right\}
$$

for a positive number $\varepsilon(<)$. It is classical that $\hat{D}$ is the envelope of holomorphy of $D$.

Lemma. Let $G$ be a complex Lie group. For any holomerphic mapping $f$ of $D$ into $G$, there is a holomorphic mapping $g$ of $\hat{D}$ into $G$ such that $g=f$ in $D$.

Proof: We may assume that $G$ is connected. We introduce in $\mathcal{H}(D, G)$ the compact-open topology $\tau_{0}$ and let $\mathcal{H}=\left(\mathcal{H}(D, G), \tau_{0}\right)$. As $D$ is analytically contractible to a point, $\mathcal{H}$ is a connected topological group. Let

$$
\begin{aligned}
& K(\delta)=\left\{z \in \mathbb{C}^{2}:\left|z_{1}\right| \leq 1+\varepsilon-\delta,\left|z_{2}\right|<1-\delta\right\} \cup \\
& \left\{z \in \mathbb{C}^{2}: 1-\varepsilon+\delta \leq\left|z_{1}\right| \leq 1+\varepsilon-\delta,\left|z_{2}\right|<1+\varepsilon-\delta\right\} \\
& \text { and } \\
& K^{\prime}(\delta)=\left\{z \in \mathbf{C}^{2}:\left|z_{j}\right|<1+\varepsilon-\delta(j=1,2)\right\}
\end{aligned}
$$

for any positive number $\delta$ with $\delta<\varepsilon$. Let $m$ be the complex dimension of $G$ and exp the exponential mapping of $\mathbb{C}^{m}$ into $G$. The mapping exp maps an open neighbourhood

$$
U=\left\{w \in \mathbb{C}^{m}:\left|w_{j}\right|<a(j=1,2, \ldots, m)\right\}
$$

of the origin in $\mathbf{C}^{m}$ biholomorphically onto an open neighbourhood $W$ of the identity element $e$ of $G$. Hence $\log :=(\exp \mid U)^{-1}$ is a biholomorphic mapping of $W$ onto $U$. We let

$$
\mathcal{V}(1)=\{h \in \mathcal{H} ; h(K(\delta)) \subset W\}
$$

Then $\mathcal{V}(1)$ is a neighbourhood of the identity element 1 of the topological group $\mathcal{H}$. Since $\mathcal{H}$ is connected, $\mathcal{H}$ is generated by $\mathcal{V}(1)$. There is a finite number $s$ of elements $f_{1}, f_{2}, \cdots, f_{s-1}$ and $f_{s}$ in $\mathcal{V}(1)$ such that

$$
f=f_{1} f_{2} \cdots f_{s}
$$


in $D$. Each $\log f_{i}$ is a holomorphic mapping of $K(\delta)$ into the polydisc $U$.

There is a holomorphic mapping $G_{i}$ of $K^{\prime}(\delta)$ into $U$ such that

$$
G_{i}=\log f_{i} \text { in } K(\delta) \cap K^{\prime}(\delta)
$$

for $j=1,2, \cdots, s$. We let

$$
g=\exp G_{1} \exp G_{2} \cdots \exp G_{s}
$$

in $K^{\prime}(\delta)$. Then $g$ is a holomorphic mapping of $K^{\prime}(\delta)$ in $G$ such that $g=f$ in $K(\delta) \cap K^{\prime}(\delta)$. Since $\delta$ is arbitrary, we obtain the Lemma by the identity theorem for holomorphic mappings. [12].

Further details and precise definitions are given in [1], [6] and

Acknowledgement The author is indebted to Professors S. Dineen, J. Kajiwara and M. Nishihara for suggesting the study of the continuation problem.

\section{References}

[1] K. Adachi, M. Suzuki and M. Yoshida, Contimuation of holomorphic mappings, with values in a complex Lie group, Pacific J. Math. 47 (1973), 1-4.

[2] H. Cartan and P. Thullen, Zur Theorie der Singularitäten der Funktionen mehrerer komplexer Verändlichen, Regularitäts- und Konvergenzbereiche, Math. Ann. 106 (1932), 617-647.

[3] F. Docquier and H. Grauert, Levisches Problem und Rungescher Satz für Teilgebiete Steinscher Mannigfaltigkeiten, Math. Ann. 140 (1960), 94-123.

[4] S. Dineen, Sheaves of holomorphic functions on infinite dimensional vector spaces, Math. Ann. 202 (1973), 337-345.

[5] L. Gruman, The Levi problem in certain infinite dimensional vector spaces, Illinois J. Math., 18 (1974), 20-26.

[6] J. Kajiwara, L. Li, M. Nishihara and M. Yoshida, Continuation of holomorphic sections of holomorphic fiber bundles over domains of infinite dimensional spaces equipped with the finite open topology, Fukuoka Univ. Sci. Rep. 23 (2) (1993), 69-79.

[7] J. Kajiwara, On the limit of a monotonous sequence of Cousin's domain J. Math. Soc. Japan 17 (1965), 36-46.

[8] J. Kajiwara and H. Kazama, Oka's principle for relative cohomology set, Mem. Fac. Sci. Kyushu Univ. 23 (1969), 33-70.

[9] J. Kajiwara and K. H. Shon, Continuation and vanishing theorem for cohomology of infinite dimensional spaces, Kyonbuk J. Math. (1993).

[10] B. Malgrange, On the Theory of Functions of Several Complex Variables. Tata Inst. Fund. Res. Bombay 13: (1958)

[11] J. Mujica, Complex Analysis in Banach Spaces. North-Holland Math. Studies 120: (1986).

[12] M. Nishihara, J. Kajiwara, L. Li and M. Yoshida, On the Levi problem for holomorphic mappings of Riemann domains over infinite dimensiona spaces into a complex Lie group, Res. Bull. Fukuoka Inst. Tech. 26 (2) $(1994), 151-161$.

[13] M. Schottenloher, The Levi problem for domains spread over locally convex spaces with a finite dimensional Schauder decomposition, Ann. Inst. Fourier 26 (1976), 207-237.

Mamoru Yoshida

Department of Mathematics

Faculty of Science

Fukuoka University

Fukuoka 814-80

Japan 